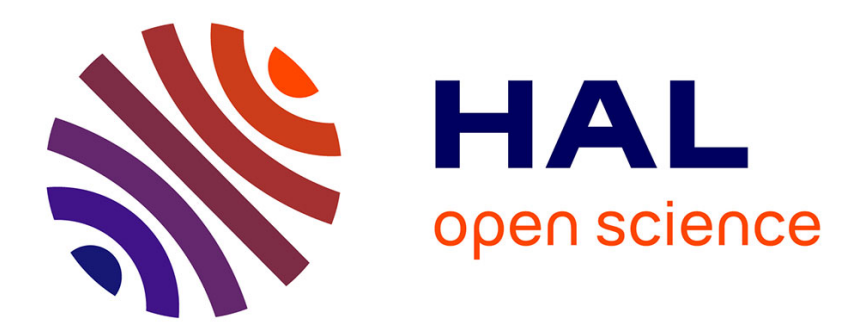

\title{
A new color filter array with optimal sensing properties Laurent Condat
}

\section{To cite this version:}

Laurent Condat. A new color filter array with optimal sensing properties. Actes de IEEE ICIP, Nov 2009, Le Caire, Egypt. pp.457 - 460, 10.1109/ICIP.2009.5414383 . hal-00814058

\section{HAL Id: hal-00814058 https://hal.science/hal-00814058}

Submitted on 16 Apr 2013

HAL is a multi-disciplinary open access archive for the deposit and dissemination of scientific research documents, whether they are published or not. The documents may come from teaching and research institutions in France or abroad, or from public or private research centers.
L'archive ouverte pluridisciplinaire HAL, est destinée au dépôt et à la diffusion de documents scientifiques de niveau recherche, publiés ou non, émanant des établissements d'enseignement et de recherche français ou étrangers, des laboratoires publics ou privés. 


\title{
A NEW COLOR FILTER ARRAY WITH OPTIMAL SENSING PROPERTIES
}

\author{
Laurent Condat ${ }^{*}$ \\ GREYC \\ 6 Bd du Maréchal Juin \\ 14050 CAEN Cedex, France
}

\begin{abstract}
We propose a new color filter array (CFA) with optimal characteristics for the acquisition of color scenes, since the luminance and chrominance information is encoded in the mosaicked image with the best achievable robustness to aliasing and noise. Our $2 \times 3$ pattern is based on the paradigm recently introduced by Hirakawa et al. [1], which focuses on the spectral properties of CFAs. Moreover, these superior properties are fully exploited by a simple, linear and efficient demosaicking method.
\end{abstract}

Index Terms - Color filter array (CFA), demosaicking, spatiospectral sampling, noise sensitivity.

\section{INTRODUCTION}

In digital still and video cameras, a color filter array (CFA) is overlaid on the sensor, in order to encode the color information as high frequency content in the mosaicked image $[2,1]$. Hence, the demosaicking task, which consists in reconstructing a color image with its three R,G,B channels, essentially amounts to appropriately assigning the frequency content of the mosaicked image to these three channels [2, 3]. Based on this observation, Hirakawa et al. recently reformulated the problem of CFA design as the minimization of aliasing between the baseband luminance and the chrominance modulated at high frequencies in the Fourier domain [1]. A similar methodology was proposed in [4]. In fact, most of the demosaicking artifacts which occur with the popular Bayer CFA originate from aliasing between the luminance and the R/B chrominance bands of the color scene, since the latter is modulated on the vertical and horizontal axes of the frequency plane, where most luminance energy is present [2]. Therefore, the CFA has to be designed so that its chrominance is modulated farther away from the origin of the frequency plane, to maximize the robustness to aliasing. However, many parameters are left free with this framework and the problem of noise sensitivity is not adressed. In this article, we exhibit the admissible pattern having maximum chrominance energy for a given luminance sensitivity. This provides the best achievable signal-to-noise ratio when the mosaicked image is contaminated by sensor noise.

The paper is organized as follows. In Section 2, we express the design problem in the Fourier domain. In Section 3, we enforce some constraints to ensure optimal sensitivity properties for the CFA. A new $2 \times 3$ pattern shows up as the unique solution. In Section 4, we present a simple and efficient linear demosaicking method, which fully exploits the spectral characteristics of our CFA.

${ }^{*}$ Part of this work was performed during the stay of the author in the Helmholtz Zentrum München, supported by the Marie Curie Excellence Team Grant MEXT-CT-2004-013477, Acronym MAMEBIA, funded by the European Commission. Contact: laurent.condategreyc.ensicaen.fr.

\section{SPECTRAL CHARACTERIZATION OF CFAS}

In this article, boldface quantities denote vectors, e.g. $\mathbf{k}=$ $\left[k_{1}, k_{2}\right]^{\mathrm{T}} \in \mathbb{Z}^{2}$ and $\mathbf{0}=[0,0]^{\mathrm{T}}$. We define a CFA as a color image $\mathbf{c f a}=(\mathbf{c f a}[\mathbf{k}])_{\mathbf{k} \in \mathbb{Z}^{2}}$, where $\mathbf{c f a}[\mathbf{k}]=$ $\left[\mathrm{cfa}^{R}[\mathbf{k}], \mathrm{cfa}^{G}[\mathbf{k}], \mathrm{cfa}^{B}[\mathbf{k}]\right]^{\mathrm{T}} \in[0,1]^{3}$ is the color value in the $\mathrm{R}, \mathrm{G}, \mathrm{B}$ basis of the filter centered at the location $\mathrm{k}$. The color values, constrained to lie in $[0,1]$ for physical realizability, correspond to opacity rates: the white color $[1,1,1]^{\mathrm{T}}$ stands for a transparent filter. We only consider periodic CFAs defined on the square lattice, and we denote by $N_{1} \times N_{2}$ the size of its smallest repeating pattern.

We define the color image $\mathbf{i m}=(\mathbf{i m}[\mathbf{k}])_{\mathbf{k} \in \mathbb{Z}^{2}}$ as the ground truth to be estimated by the demosaicking process. The mosaicked image $v=(v[\mathbf{k}])_{\mathbf{k} \in \mathbb{Z}^{2}}$ is such that $v[\mathbf{k}]=\mathbf{i m}[\mathbf{k}]^{\mathrm{T}} \mathbf{c f a}[\mathbf{k}], \forall \mathbf{k} \in \mathbb{Z}^{2}$. In practice, a random term modeling the effect of noise has to be added to this model [5].

It is well known that in natural images, the R,G,B components are not independent $[6,7]$. Thus, we define the orthonormal basis corresponding to luminance, red/blue and green/magenta chrominances, as $\mathbf{L}=\frac{1}{\sqrt{3}}[1,1,1]^{\mathrm{T}}, \mathbf{C}_{1}=\frac{1}{\sqrt{2}}[1,0,-1]^{\mathrm{T}}, \mathbf{C}_{2}=$ $\frac{1}{\sqrt{6}}[-1,2,-1]^{\mathrm{T}}$. We denote by $u^{L}, u^{C_{1}}$, and $u^{C_{2}}$ the components of a color signal $\mathbf{u}$ in this basis. They can be considered statistically independent for natural images [6].

In order to analyse the properties of the Bayer CFA, Alleysson et al. showed that the mosaicked image $v$ can be interpreted, in the Fourier domain, as the sum of the luminance and chrominance components of the color image im, moved at different locations of the frequency plane [2]. This characterization can be extended to every CFA, by simply writing cfa as the sum of its Fourier components:

$$
\begin{aligned}
\operatorname{cfa}^{X}[\mathbf{k}]= & \sum_{n_{1}=\left\lfloor\frac{N_{1}-1}{2}\right\rfloor}^{\left\lfloor\frac{N_{1}}{2}\right\rfloor} \sum_{n_{2}=0}^{\left\lfloor\frac{N_{2}}{2}\right\rfloor} \alpha_{\mathbf{n}}^{X} \cos \left(\frac{2 \pi n_{1}}{N_{1}} k_{1}+\frac{2 \pi n_{2}}{N_{2}} k_{2}\right)+ \\
& \beta_{\mathbf{n}}^{X} \sin \left(\frac{2 \pi n_{1}}{N_{1}} k_{1}+\frac{2 \pi n_{2}}{N_{2}} k_{2}\right)
\end{aligned}
$$

for every $X \in\left\{L, C_{1}, C_{2}\right\}$ and $\mathbf{k} \in \mathbb{Z}^{2}$. So, designing cfa amounts to choosing its $3 N_{1} N_{2}$ Fourier coefficients $\alpha_{\mathbf{n}}^{X}$ and $\beta_{\mathbf{n}}^{X}$ appropriately. For this, we express the Fourier transform $\hat{v}(\boldsymbol{\omega})=$ $\sum_{\mathbf{k} \in \mathbb{Z}^{2}} v[\mathbf{k}] e^{-j \boldsymbol{\omega}^{\mathrm{T}} \mathbf{k}}$ in function of the Fourier transforms of the components of im:

$\hat{v}(\boldsymbol{\omega})=\sum_{X \in\left\{L, C_{1}, C_{2}\right\}} \sum_{n_{1}=\left\lfloor\frac{N_{1}-1}{2}\right\rfloor}^{\left\lfloor\frac{N_{1}}{2}\right\rfloor} \sum_{n_{2}=0}^{\left\lfloor\frac{N_{2}}{2}\right\rfloor}$,

$\frac{\alpha_{\mathbf{n}}^{X}}{2}\left(\widehat{\operatorname{im}^{X}}\left(\boldsymbol{\omega}+\left[\frac{2 \pi n_{1}}{N_{1}}, \frac{2 \pi n_{2}}{N_{2}}\right]^{\mathrm{T}}\right)+\widehat{\operatorname{im}^{X}}\left(\boldsymbol{\omega}-\left[\frac{2 \pi n_{1}}{N_{1}}, \frac{2 \pi n_{2}}{N_{2}}\right]^{\mathrm{T}}\right)\right)+$ $\frac{\beta_{\mathbf{n}}^{X}}{2 j}\left(\widehat{\operatorname{im}^{X}}\left(\boldsymbol{\omega}+\left[\frac{2 \pi n_{1}}{N_{1}}, \frac{2 \pi n_{2}}{N_{2}}\right]^{\mathrm{T}}\right)-\widehat{\operatorname{im}^{X}}\left(\boldsymbol{\omega}-\left[\frac{2 \pi n_{1}}{N_{1}}, \frac{2 \pi n_{2}}{N_{2}}\right]^{\mathrm{T}}\right)\right)$, 
for every $\boldsymbol{\omega} \in \mathbb{R}^{2}$. Hence, whatever the CFA, $\hat{v}$ is the sum of the luminance and chrominance components $\widehat{\mathrm{im}^{X}}$, replicated at the sites of the dual lattice induced by the periodicity of the pattern. This is essentially the task of demosaicking to separate these components back. Given this observation, Hirakawa et al. had the ingenious idea to directly design the CFA in the Fourier domain, so that the components $\widehat{\mathrm{im}^{X}}$ are well separated in $\hat{v}$ [1]. In their framework, the baseband luminance is at the origin $\left(\mathrm{cfa}^{L}[\mathbf{k}]=\alpha_{\mathbf{0}}^{L}\right.$, in (1) ) and the chrominance is modulated far away from it, on the boundaries of the Nyquist band (for this, we assume $N_{1}=2$ ) and far away from the axes of the frequency plane [1]. This constrains the coefficients $\alpha_{\mathbf{n}}^{X}, \beta_{\mathbf{n}}^{X}$ to some extent, but the question of further defining the remaining degrees of freedom is left open. This is the aim of this work to show how to tune these parameters to obtain a CFA with optimal sensitivity characteristics.

\section{A NEW CFA WITH OPTIMAL SENSITIVITY CHARACTERISTICS}

We now construct our new CFA step by step, by enforcing several design criteria in the frequency domain.

First, in order to maximally reduce the overlaps between the channels, we impose the chrominance to be shifted at only one frequency $\pm \boldsymbol{\omega}_{0}$, with $\boldsymbol{\omega}_{0}=\left[\pi, \omega_{0}\right]^{\mathrm{T}}$ and $\omega_{0}=2 \pi n_{2} / N_{2}$ for some integer $n_{2} \in\left(1,\left\lfloor\left(N_{2}-1\right) / 2\right\rfloor\right)$ relatively prime with $N_{2}$. Hence, the luminance information is concentrated around $\mathbf{0}$ in $\hat{v}$, while the chrominance information is around $\pm \boldsymbol{\omega}_{0}$. Moreover, the two chrominance bands should be orthogonal, so that they can be optimally separated during demosaicking. In comparison with the designs in [1], whith the chrominance spread at several frequencies, the risk of inter-chrominance aliasing is drastically reduced. We also impose that the carrier waves of the two chrominance channels have the same magnitude, so that the color discrimination of the CFA is the same for every color, without privileged chrominance axis. Hence, the two carrier waves are sines in quadrature and we obtain the form of the CFA as

$$
\begin{aligned}
\operatorname{cfa}^{L}[\mathbf{k}] & =\gamma^{L}, \\
\operatorname{cfa}^{C}[\mathbf{k}] & =\gamma^{C}(-1)^{k_{1}} \sqrt{2} \sin \left(\omega_{0} k_{2}-\varphi\right), \\
\operatorname{cfa}^{C}[\mathbf{k}] & =\gamma^{C}(-1)^{k_{1}} \sqrt{2} \cos \left(\omega_{0} k_{2}-\varphi\right),
\end{aligned}
$$

for every $\mathbf{k} \in \mathbb{Z}^{2}$, where we introduced the luminance gain $\gamma^{L}$, the chrominance gain $\gamma^{C}$ and the phase $\varphi$ which are, at this point, free degrees of freedom. Note that $\omega_{0}$ must be different from 0 and $\pi$. Therefore, $N_{2} \geq 3$.

We now remark that replacing each value $\mathrm{cfa}^{X}[\mathbf{k}]$ by $1-$ $\mathrm{cfa}^{X}[\mathbf{k}]$ for every $X \in\{R, G, B\}$ does not change the spectral properties of the CFA, apart from $\gamma^{L}$ which is changed into $\sqrt{3}-\gamma^{L}$. Therefore, this operation can be done to ensure that $\gamma^{L} \geq \sqrt{3} / 2$, since the light sensitivity of a CFA is one of its most crucial characteristics and should be maximal. However, we have to fix $\gamma^{L}=\sqrt{3} / 2$, since increasing this parameter also reduces $\gamma^{C}$, which is already small for this value of $\gamma^{L}$. A higher value of $\gamma^{L}$ would yield a much higher amplification of the noise in the chrominance bands than in the luminance band of the demosaicked image.

The cornerstone of the design is now the maximization of $\gamma^{C}$, so that the CFA has the best color discrimination capabilities, for reduced luminance/chrominance aliasing and lower sensitivity to noise. After some calculations omitted by lack of space, we obtain
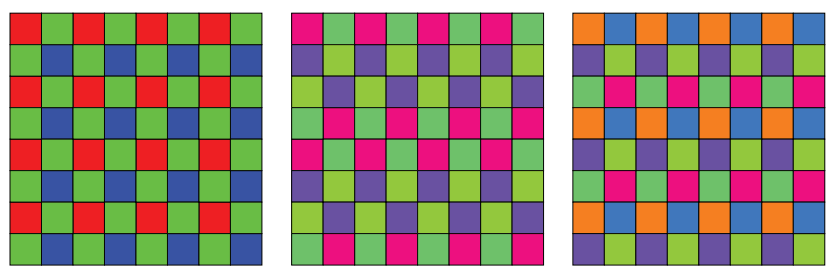

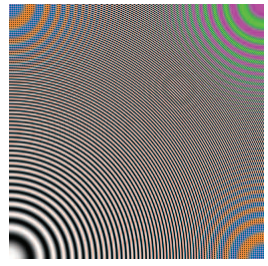

Bayer

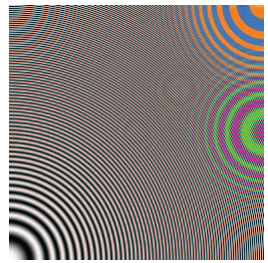

Hirakawa et al. [1]

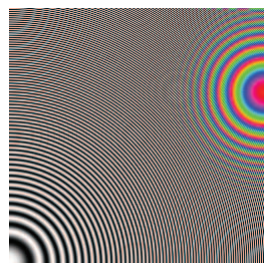

Proposed
Fig. 1. The thee CFAs considered in this work (top) and the respective results of our demosaicking method on a grayscale synthetic zoneplate pattern (bottom), to illustrate their spectral properties.

that, for fixed $N_{2}$ and $n_{2}$, the desired values in (3)-(4) are

$$
\gamma^{C}=\frac{\sqrt{3}}{4 \cos (\varphi)}, \quad \varphi=\frac{\pi}{\operatorname{lcm}\left(6, N_{2}\right)},
$$

where $1 \mathrm{~cm}$ denotes the least common multiple. Consequently, the value $N_{2}=3$ (therefore, $n_{2}=1$ ) maximizes the chrominance gain $\gamma^{C}$ (the other solution $N_{2}=6, n_{2}=1$ is not interesting, since its modulating frequency $[\pi, \pi / 3]^{\mathrm{T}}$ is closer to $\mathbf{0}$ ).

The obtained $2 \times 3$ pattern, depicted in Fig. 1, has a small number (six) of distinct filters, with the colors $\left[1,0, \frac{1}{2}\right],\left[1, \frac{1}{2}, 0\right]$, $\left[0,1, \frac{1}{2}\right],\left[0, \frac{1}{2}, 1\right],\left[\frac{1}{2}, 0,1\right]$ and $\left[\frac{1}{2}, 1,0\right]$. Its modulation frequency $\boldsymbol{\omega}_{0}=[\pi, 2 \pi / 3]^{\mathrm{T}}$ is ideally located. It is far away from the origin and the axes of the frequency plane, but not too close to $[\pi, \pi]^{\mathrm{T}}$, in which case the risk of inter-chrominance aliasing would be increased (because of the overlap with the replica at $\left[\pi, 2 \pi-\omega_{0}\right]^{\mathrm{T}}$ ). The gains, which control the sensitivity to noise, are $\gamma^{L}=\sqrt{3} / 2$ and $\gamma^{C}=1 / 2$. Moreover, the pattern does not depend on the choice of the chrominance basis $\mathbf{C}_{1}, \mathbf{C}_{2}$, only used for the calculations. Our new CFA provides much better characteristics than the Bayer CFA for photography: its higher light sensitivity (higher $\gamma^{L}$ ) allows to reduce the exposure time (for less blur due to shake of the camera), to increase the aperture (for increased depth-of-field, hence less out-of-focus blur), or to use a lower ISO setting for a less destructive subsequent denoising process. It offers a better robustness to both luminance/chrominance aliasing and noise, as illustrated in Sect. 5.

\section{DEMOSAICKING STRATEGY}

A simple linear demosaicking algorithm naturally follows from the characteristics of our CFA in the Fourier domain. It consists in separating the frequency content of the mosaicked image $v$ into the luminance and chrominance channels of the reconstructed image. For this, we first estimate the chrominance and then subtract it to $v$ to obtain the luminance. The chrominance is obtained by re-modulating $v$ so that the chrominance is shifted in the low frequency area, and then applying a low-pass filter. So, the complexity of the demosaicking process is essentially limited to two convolutions! In addition, they use the same filter and can be performed in parallel. The demosaicking method proposed consists in the following steps, where we denote by dem the demosaicked image. 


\begin{tabular}{c|cc||ccc|}
$\begin{array}{c}\text { Demosa. } \\
\text { method }\end{array}$ & \multicolumn{2}{|c||}{$\begin{array}{c}\text { Non-linear methods } \\
{[3]}\end{array}$} & \multicolumn{3}{c|}{$\begin{array}{c}\text { Proposed linear method } \\
\text { with } 9 \times 9 \text { filters }\end{array}$} \\
\hline CFA & Bayer & Bayer & Bayer & {$[1]$} & Ours \\
\hline 1 & 10.12 & 11.45 & 12.93 & $\mathbf{6 . 5 3}$ & 7.12 \\
2 & 6.80 & 5.75 & 7.92 & $\mathbf{6 . 0 1}$ & 7.07 \\
3 & 10.56 & 10.14 & 13.14 & $\mathbf{1 2 . 5 8}$ & 13.38 \\
4 & 6.47 & 10.15 & 10.26 & $\mathbf{4 . 8 0}$ & 5.32 \\
5 & 4.00 & 5.02 & $\mathbf{3 . 3 4}$ & 4.59 & 4.53 \\
6 & 19.31 & 19.26 & 29.25 & $\mathbf{1 0 . 9 2}$ & 11.90 \\
7 & 3.96 & 3.38 & 5.16 & $\mathbf{3 . 8 8}$ & 4.00 \\
8 & 3.91 & 3.56 & 4.42 & $\mathbf{3 . 5 7}$ & 3.58 \\
9 & 6.80 & 7.49 & 8.74 & $\mathbf{5 . 7 6}$ & 6.14 \\
10 & 3.20 & 3.39 & 4.17 & 2.93 & $\mathbf{2 . 9 2}$ \\
11 & 19.98 & 27.03 & 20.39 & $\mathbf{1 6 . 8 7}$ & 17.90 \\
12 & 7.35 & 7.08 & 7.82 & $\mathbf{7 . 0 6}$ & 7.36 \\
13 & 2.77 & 4.92 & 5.26 & $\mathbf{2 . 1 0}$ & 2.15 \\
14 & 4.42 & 4.66 & 4.69 & $\mathbf{4 . 1 4}$ & 4.54 \\
15 & 12.05 & 13.01 & 12.42 & $\mathbf{1 1 . 1 5}$ & 11.81 \\
16 & 5.92 & 6.30 & 10.06 & $\mathbf{4 . 4 8}$ & 4.87 \\
17 & 5.92 & 5.04 & 6.11 & $\mathbf{4 . 6 4}$ & 5.05 \\
18 & 8.51 & 8.72 & 9.34 & $\mathbf{5 . 5 9}$ & 6.04 \\
19 & 9.86 & 9.00 & 10.78 & $\mathbf{8 . 4 8}$ & 8.81 \\
20 & 18.94 & 21.42 & 19.29 & $\mathbf{1 4 . 2 4}$ & 15.44 \\
\hline Mean & 8.54 & 9.25 & 10.36 & 7.02 & 7.50
\end{tabular}

Table 1. MSE for the linear demosaicking experiments using different CFAs, in the noiseless case. Image numbers correspond to [9].

1. Compute the image $v_{1}$ from $v$ by modulation with the carrier wave of the chrominance $C_{1}: v_{1}[\mathbf{k}]=$ $(-1)^{k_{1}} \sqrt{2} \sin \left(\omega_{0} k_{2}-\varphi\right) / \gamma^{C} v[\mathbf{k}]$.

2. Apply the convolution with the appropriate low-pass filter $h$ : $\operatorname{dem}^{C_{1}}=v_{1} * h$.

3. Compute the image $v_{2}$ from $v$ by modulation with the carrier wave of the chrominance $C_{2}: v_{2}[\mathbf{k}]=$ $(-1)^{k_{1}} \sqrt{2} \cos \left(\omega_{0} k_{2}-\varphi\right) / \gamma^{C} v[\mathbf{k}]$.

4. Apply the convolution with the same low-pass filter $h$ : $\operatorname{dem}^{C_{2}}=v_{2} * h$.

5. Estimate the luminance by subtraction of the remodulated chrominance: $\operatorname{dem}^{L}[\mathrm{k}]=$ $\left(v[\mathbf{k}]-\gamma^{C}(-1)^{k_{1}} \sqrt{2} \sin \left(\omega_{0} k_{2}-\varphi\right) \operatorname{dem}^{C_{1}}[\mathbf{k}]-\right.$ $\left.\gamma^{C}(-1)^{k_{1}} \sqrt{2} \cos \left(\omega_{0} k_{2}-\varphi\right) \operatorname{dem}^{C_{2}}[\mathbf{k}]\right) / \gamma^{L}$.

6. Compute $\operatorname{dem}^{R}, \operatorname{dem}^{G}, \operatorname{dem}^{B}$ by change of basis from $\operatorname{dem}^{C_{1}}, \operatorname{dem}^{C_{2}}, \operatorname{dem}^{L}$.

In practice, the values of the carrier waves should be precomputed in a look-up table of size 6 , to exploit the periodicity of the pattern. The proposed algorithm can be easily adapted to handle other CFAs, since it is an optimization of the generic approach given in $[1,10]$ to our specific CFA. The adaptation to the Bayer pattern reverts to the linear algorithm of Dubois [3]. Concerning the choice of the filter(s) $h$, we computed for every CFA the non-separable $9 \times 9$ filter(s) optimal in the least-squares sense for the test set of 20 images considered in Sect. 5, using the approach proposed by Dubois [11].

\section{PERFORMANCE ANALYSIS}

The combination of the generic linear demosaicking framework of Sect. 4 by spectral selection with the LS-optimal filters provides a fair and robust way for comparing the performances of CFAs. In this section, we compare our new CFA with the Bayer CFA and the $2 \times 4$ CFA of Hirakawa et al. [1], depicted in Fig. 1 . To visually

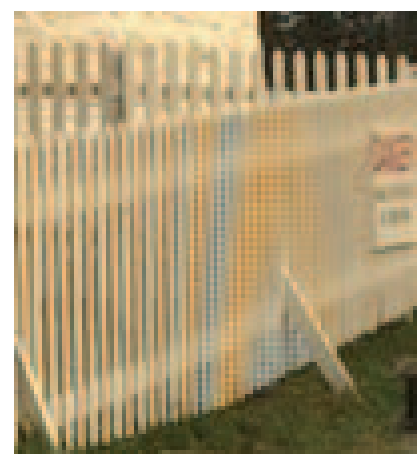

Bayer

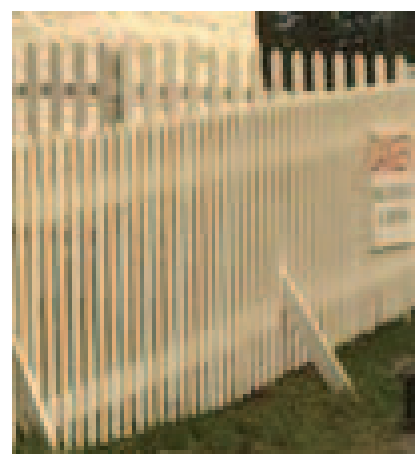

Proposed
Fig. 2. Results of our demosaicking method on a part of the Lighthouse image, with the Bayer and the proposed CFA.

illustrate the modulation of the chrominance in the Fourier domain by the CFAs, we give in Fig. 1 the images obtained when a synthetic grayscale zoneplate is mosaicked and demosaicked with the proposed method. This shows how the demosaicking method assigns the frequency content of $v$ to the luminance and chrominance bands of the demosaicked image. We can see that the CFA of Hirakawa et $a l$. is prone to aliasing between the two chrominance bands. This may be visible in demosaicked images around sharp color edges of horizontal objects.

\subsection{Evaluation in Noiseless Situations}

To validate our design, we consider the data set of 20 color images of size $768 \times 512$ used by many authors to test their methods (e.g. $[9,1])$. These images were mosaicked using the considered CFAs and demosaicked using spectral selection, as discussed in Sect. 4. Note that to simulate an acquisition with a real camera, all images were put in landscape mode. The mean squared errors (MSE) ${ }^{1}$ obtained are reported in Tab. 1. We also provide the results obtained for the Bayer CFA with two state-of-the-art non-linear demosaicking methods $[3,8]$. We observe that the MSE improvement of at most $18 \%$, obtained with the non-linear methods over our linear approach, is not so high, especially when taking into account the much higher computation cost. Moreover, the non-linear methods distort the characteristics of noise, which makes subsequent denoising a much more difficult task.

As a result, The CFA of Hirakawa et al. and ours clearly outperform the Bayer CFA. They provide visually pleasant demosaicked images without noticeable artifacts. In particular, they are free of the typical fringes effect that affects the Bayer CFA, see Fig. 2. The CFA of Hirakawa et al. slightly outperforms our new CFA in this noiseless scenario. In fact, with their CFA, the modulating frequency for the G/M chrominance $\omega_{0}=[\pi, \pi / 2]^{\mathrm{T}}$ is closer to zero, but with two times less energy around $\boldsymbol{\omega}_{0}$, since the second chrominance band is modulated at $[\pi, \pi]^{\mathrm{T}}$. Therefore, their CFA is slightly less prone to luminance/chrominance aliasing.

\subsection{Evaluation in Noisy Situations}

In real acquisition conditions, the mosaicked image is corrupted by sensor noise, amplified by the analog gain applied (ISO setting of

\footnotetext{
${ }^{1}$ In this article, like in [1], we do not take into account the first and las five rows and columns of the demosaicked images for the computation of the MSE, to get rid of issues at the boundaries.
} 


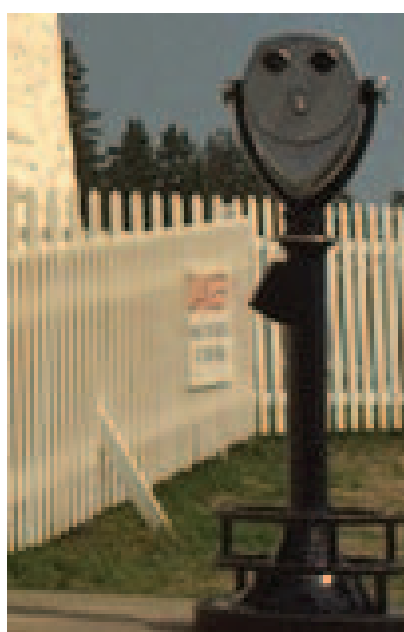

Original

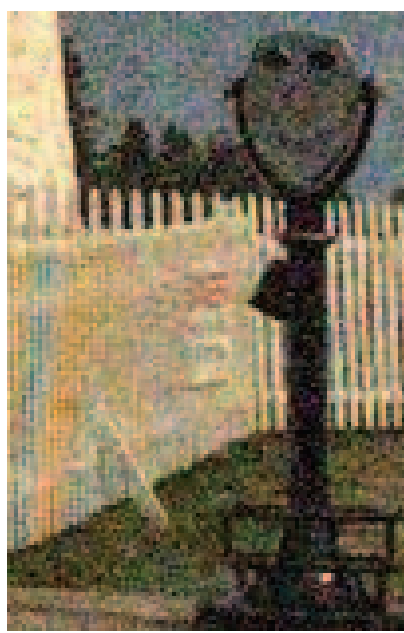

Bayer

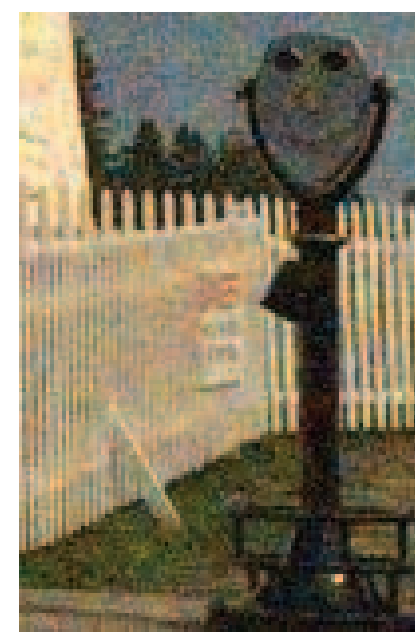

Hirakawa et al.

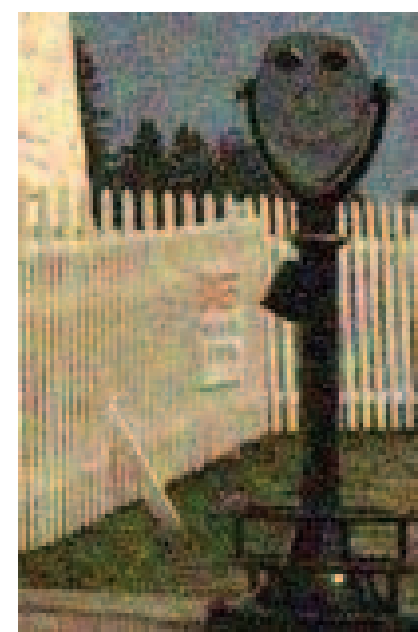

Proposed

Fig. 3. Results of our demosaicking method used with the three CFAs depicted in Fig. 1, on a part of the Lighthouse image. The mosaicked image is corrupted by additive white Gaussian noise of standard dev. $\sigma=20$ and no denoising process is applied.

the camera) [5]. We give in Fig. 3 examples of demosaicked images, when the mosaicked image is corrupted by additive white Gaussian noise. The linearity of the demosaicking process makes the analytical description of its effects on noise rather simple, but we omit it by lack of space. As can be visually observed, most of the demosaicked noise is concentrated in the luminance ( $86 \%$ of the noise energy with our CFA and our filter $h$ ). The level of luminance noise is lower with the CFA of Hirakawa et al. and ours than with the Bayer CFA, since $\gamma^{L}$ is higher.

With the CFA of Hirakawa, the two chrominance gains are different $\left(\gamma^{C_{1}}=\sqrt{2} / 4, \gamma^{C_{2}}=\sqrt{6} / 4\right)$. This means that the noise is more amplified in the $\mathrm{B} / \mathrm{R}$ band than in the G/M band after demosaicking. Our CFA is free of this issue. The amplification factor for the noise energy in the chrominance of the demosaicked image is $1 /\left(\gamma^{C_{1}}\right)^{2}+1 /\left(\gamma^{C_{1}}\right)^{2}$; that is, $32 / 3$ vs. 8 for our CFA (in other words, $33 \%$ more chrominance noise). This advantage of our CFA over the one of Hirakawa should largely counterbalance the slightly lower performances in the ideal noiseless scenario.

\section{CONCLUSION}

We redefined the problem of CFA design as the maximization of the energy of the color scene captured in the mosaicked image, through the choice of the luminance and chrominance gains of the CFA, within the framework of spectral separation proposed by Hirakawa $e t$ al. [1]. The proposed $2 \times 3 \mathrm{CFA}$, solution to this problem, provides optimal robustness to both aliasing and noise. Moreover, its spectral properties are fully exploited by a simple, linear and fast demosaicking strategy. The design of efficient joint demosaicking/denoising strategies will be investigated in future works. Also, the design of the spectral sensitivity functions of the six colors defining the new CFA has to be addressed, for instance with the methodology in [12].

\section{REFERENCES}

[1] K. Hirakawa and P. J. Wolfe, "Spatio-spectral color filter array design for optimal image recovery," IEEE Trans. Image Processing, vol. 17, no. 10, pp. 1876-1890, Oct. 2008.
[2] D. Alleyson, S. Susstrunk, and J. Herault, "Linear demosaicing inspired by the human visual system," IEEE Trans. Image Processing, vol. 14, no. 4, pp. 439-449, Apr. 2005.

[3] E. Dubois, "Frequency-domain methods for demosaicking of Bayer-sampled color images," IEEE Signal Processing Lett., vol. 12, no. 12, pp. 847-850, Dec. 2005.

[4] Y. Li, P. Hao, and Z. Lin, "Color filter arrays: A design methodology," Dept of Computer Science, Queen Mary, University of London, Res. Rep. 08-03, May 2008.

[5] A. Foi, M. Trimeche, V. Katkovnik, and K. Egiazarian, "Practical poissonian-gaussian noise modeling and fitting for singleimage raw-data," IEEE Trans. Image Processing, vol. 17, no. 10, pp. 1737-1754, Oct. 2008.

[6] Y. Hel-Or, "The canonical correlations of color images and their use for demosaicing," HP Laboratories Israel, Tech. Rep. HPL-2003-164R1, Feb. 2004.

[7] B. K. Gunturk, J. Glotzbach, Y. Altunbasak, R. W. Schaffer, and R. M. Mersereau, "Demosaicking: Color filter array interpolation," IEEE Signal Processing Mag., vol. 22, no. 1, pp. 44-54, Jan. 2005.

[8] L. Nai-Xiang, C. Lanlan, T. Yap-Peng, and V. Zagorodnov, "Adaptive filtering for color filter array demosaicking," IEEE Trans. Image Processing, vol. 16, no. 10, pp. 2515-2525, 2007.

[9] B. K. Gunturk, Y. Altunbasak, and R. M. Mersereau, "Color plane interpolation using alternating projections," Proc. IEEE, vol. 11, no. 9, pp. 997-1013, Sept. 2002.

[10] K. Hirakawa and P. Wolfe, "Second-generation color filter array and demosaicking designs," in Proc. of SPIE VCIP, vol. 6822, 2008.

[11] E. Dubois, "Filter design for adaptive frequency-domain Bayer demosaicking," in Proc. of IEEE ICIP, 2006, pp. 2705-2708.

[12] M. Parmar and S. J. Reeves, "Selection of optimal spectral sensitivity functions for color filter arrays," in Proc. of IEEE ICIP, Oct. 2006. 